\title{
Monopoles and Spectral Curves for Arbitrary Lie Groups
}

\author{
M. K. Murray \\ The Mathematical Institute, 24-29 St. Giles, Oxford OX1 3LB, England
}

\begin{abstract}
The definition of the spectral curve of a monopole is extended to any connected, compact, simple Lie group $K$. It is found there are rank $K$ curves whose degrees are related to the topological weights of the monopole.
\end{abstract}

\section{Introduction}

A lot is now known about static monopoles in Euclidean three-space for the gauge group $\mathrm{SU}(2)[5,7,11,13,14]$. These are solutions of the Bogomolny equations, in the Bogomolny-Prasad-Sommerfield (BPS) limit and are classified by an integer $k$, the topological weight. For $k=1$ the only monopoles are all translates of the spherically symmetric Prasad-Sommerfield monopole [10]. For higher $k$ axially symmetric solutions have been constructed $[14,12,5]$ and recently shown to be regular [8]. The existence of a large number of solutions for each $k$ with no special symmetry has been shown by Taubes [13] and Weinberg has shown that they depend on $4 k-1$ parameters [15].

By using the "twistor methods" of Penrose, Ward and Atiyah, Hitchin [7] has shown that each monopole has associated to it a real, algebraic curve of degree $2 k$, the spectral curve, from which the monopole can be reconstructed.

For groups $K$ larger than $\mathrm{SU}(2)$ the monopoles are classified by $r$ topological weights $\left(m_{1}, \ldots, m_{r}\right)$, where $r$ is the rank of $K$. Taubes has shown [13] that there exist monopoles for any $K$ and $\left(m_{1}, \ldots, m_{r}\right)$ when all the $m_{i}$ are non-negative, and Weinberg has calculated the number of parameters that these solutions depend upon [15].

The same twistor methods can be applied to these monopoles, and we show that there exist $r$ spectral curves $S_{1}, \ldots, S_{r}$ naturally associated to the vertices of the Dynkin diagram. Again the curves have degree $2 m_{i}$. If the intersections $S_{i} \cap S_{j}$ are finite whenever $i$ and $j$ are joined on the Dynkin diagram (if the curves are reducible $S_{i} \cap S_{j}$ may contain a component), then the monopole can be reconstructed from the spectral curves. 
All the algebraic geometry needed to prove the results mentioned will appear at a later date (see also Sect. 5), but it is hoped that the results are of interest in themselves, particularly in light of a recent article [11] by Nahm which, using an entirely different method associates $n-1$ curves to an $\mathrm{SU}(n)$ monopole.

\section{The SU(2) Case}

A monopole, for a group $K$, is a gauge potential $A=\sum_{i=1}^{3} A_{i} d x^{i}$ and a Higgs field $\Phi$, defined on $\mathbb{R}^{3}$ and taking values in $L K$, the Lie algebra of $K$, which satisfy the Bogomolny equations and the Bogomolny-Prasad-Sommerfield (BPS) boundary conditions.

The Bogomolny equations are

$$
* F=D \Phi
$$

or

$$
\sum_{j, k=1}^{3} \varepsilon_{i j k} F_{j k}=D_{i} \Phi
$$

for $i=1,2,3$, where $D_{i}=\partial_{i}+A_{i}$ is the covariant derivative, and $F_{j k}=\partial_{j} A_{k}-\partial_{k} A_{j}$ $+\left[A_{j}, A_{k}\right]$ is the gauge field.

For SU(2) the BPS boundary conditions are that

$$
\operatorname{tr} \Phi^{2}=1-\frac{k}{2 r}+O\left(\frac{1}{r^{2}}\right)
$$

This means that $\Phi^{\infty}$, the Higgs field at infinity, takes its value in the two-sphere inside $L S U(2) \cong \mathbb{R}^{3}$. As this two-sphere is an orbit of the adjoint action of $S U(2)$, the appropriate boundary conditions for a group $K$ are that in some gauge,

$$
\Phi=\Phi^{\infty}+\psi \frac{1}{r}+O\left(\frac{1}{r^{2}}\right)
$$

where $\Phi^{\infty}$ takes its values in an orbit of $K$ on $L K$ and $\psi: S_{\infty}^{2} \rightarrow L K$. If $\Phi^{\infty}(N)=\lim _{t \rightarrow \infty} \Phi(0,0, t)$ and $H=\left\{k \in K \mid \operatorname{ad} k \Phi^{\infty}(N)=\Phi^{\infty}(N)\right\}$, then the orbit is of the form $K / H$. We are restricting ourselves to the case where $H=T$ a maximal torus in $K$. For $K=\mathrm{SU}(n), \mathrm{Sp}(n), \mathrm{SO}(2 n)$ and $\mathrm{SO}(2 n+1)$, the maximal tori are [1],

$$
\begin{aligned}
& K=\mathrm{SU}(n) \quad T=\text { diagonal matrices, } \\
& K=\operatorname{Sp}(n) \quad T=\text { diagonal matrices, } \\
& K=\mathrm{SO}(2 n) \quad T=\text { matrices of the form } \\
& {\left[\begin{array}{ccc}
D_{1} & & 0 \\
0 & \ddots & D_{n}
\end{array}\right], \quad \text { where } \quad D_{i} \in \operatorname{SO}(2),}
\end{aligned}
$$




$$
\begin{gathered}
K=\operatorname{SO}(2 n+1) \quad T=\text { matrices of the form } \\
{\left[\begin{array}{ll}
X & 0 \\
0 & 1
\end{array}\right] \text { for } X \in \operatorname{SO}(2 n) .}
\end{gathered}
$$

For $\mathrm{SU}(n)$ this means that $\Phi^{\infty}$ has distinct eigenvalues.

If we write the Bogomolny equations in spherical polar coordinates, in a gauge with $A_{r}=\sum_{i=1}^{3} x^{i} A_{i}=0$, we have for the $r$-component

$$
\partial_{r} \Phi=\frac{1}{r^{2}} \frac{1}{\sin \theta} F_{\theta \phi}
$$

Differentiating (4), we see that if we let

$$
\frac{1}{\sin \theta} F_{\theta \phi}={ }^{*} F_{\theta \phi}^{\infty}+O\left(\frac{1}{r}\right), \text { then } \Phi=\Phi^{\infty}-{ }^{*} F^{\infty}\left(\frac{1}{r}\right)+O\left(\frac{1}{r^{2}}\right) \text {. }
$$

As an example, for $\mathrm{SU}(n)$ we have

$$
\Phi=i \operatorname{diag}\left(\lambda_{1}, \ldots, \lambda_{n}\right)-\frac{i}{2 r} \operatorname{diag}\left(k_{1} \ldots, k_{n}\right)+O\left(\frac{1}{r^{2}}\right),
$$

with $\sum_{i=1}^{n} \lambda_{i}=\sum_{i=1}^{n} k_{i}=0, \lambda_{1}>\ldots>\lambda_{n}$ and where $\operatorname{diag}\left(\lambda_{1}, \ldots, \lambda_{n}\right)$ is the diagonal matrix with diagonal entries $\lambda_{1}, \ldots, \lambda_{n}$ and for topological reasons [6] $k_{1}, \ldots, k_{n}$ are integers.

The construction used to define the spectral curve is as follows [7]. The twistor space $T$ is defined to be the set of all orientated lines in $\mathbb{R}^{3}$. If $\gamma$ is such a line, we let $-\gamma$ denote the line with opposite orientation. For any such line $\gamma$ there is a unique pair of vectors ( $\mathbf{u}, \mathbf{v})$ satisfying $\mathbf{u} \cdot \mathbf{u}=1, \mathbf{u} \cdot \mathbf{v}=0$ and such that $\gamma=\{t \mathbf{u}+\mathbf{v} \mid t \in \mathbb{R}\}$ with $\mathbf{u}$ pointing along the direction of orientation. But this also describes a tangent vector $\mathbf{v}$ to $S^{2}$ at $\mathbf{u}$, so we see that $T$ is also the tangent bundle to the sphere $S^{2}$.

The two-sphere $S^{2}$ can be given complex coordinates in the standard way by projecting stereographically from some point $N \in S^{2}$. If $z$ is the coordinate of some point, $p$, a tangent vector is of the form $w \frac{\partial}{\partial z}$, so for each line $\gamma$ we can define coordinates $(w, z)$. This, of course, doesn't work for lines parallel to the vector $N$, but if we project from the antipode of $N$ we can give these lines coordinates and on the area of overlap one set of coordinates is a complex analytic function of the other.

Now for the case of SU(2), given the monopole $\left(A_{i}, \Phi\right)$ Hitchin [7] defines a vector space $E(\gamma)$ for each line $\gamma$, which is the two dimensional complex space of solutions of the equation

$$
\left(u^{i} \partial_{i}+u^{i} A_{i}(x)-i \Phi(x)\right) v(x)=0,
$$

where $x \in \gamma=(\mathbf{u}, \mathbf{v})$ and $v(x)$ is $\mathbb{C}^{2}$ valued. If $t$ is a parameter along the line, this equation can be abbreviated to

$$
\left(D_{t}-i \Phi(t)\right) v(t)=0
$$


Using the fact that $\mathrm{SU}(2)=\mathrm{Sp}(1)$, the group of unit quaternions, a map $\sigma: E(\gamma) \rightarrow E(-\gamma)$ can be defined satisfying $\sigma^{2}=-1$ and called the quaternionic structure.

This collection of vector spaces $E=E(\gamma)$ can be given the structure of a holomorphic vector bundle and [7] shows that this is equivalent to a solution of the Bogomolny equations. If, in addition, the solution satisfies the BPS boundary conditions, the spectral curve $S$ can be defined.

To do this the boundary condition (3) is substituted into the differential equation to give

$$
\frac{d v}{d t}+\left[\begin{array}{lc}
\lambda-k / 2 t & 0 \\
0 & -\lambda+\frac{k}{2 t}
\end{array}\right] v+O\left(\frac{1}{t^{2}}\right) v=0
$$

in a gauge where $\sum_{i=1}^{3} u^{i} A_{i}=0$. Using a result from [4], this means that there are solutions $v_{1}(t), v_{2}(t)$ to $(15)$ satisfying

$$
v_{1}(t) t^{-k / 2} e^{\lambda t} \rightarrow\left(\begin{array}{l}
1 \\
0
\end{array}\right) \text { and } v_{2}(t) t^{k / 2} e^{-\lambda t} \rightarrow\left(\begin{array}{l}
0 \\
1
\end{array}\right)
$$

as $t \rightarrow+\infty$. So there is a 1-dimensional space $L^{+}(\gamma) \subset E(\gamma)$ of solutions decaying at infinity. Letting $s=-t$ we see that there is also a 1-dimensional space $L^{-}(\gamma)$ of solutions decaying at negative infinity. Then the spectral curve $S$ is the set of $\gamma$ such that $L^{+}(\gamma)=L^{-}(\gamma)$.

The curve $S$ has two important properties. Firstly we can deduce easily that $\sigma\left(L^{+}(\gamma)\right)=L^{-}(-\gamma)$, and so $\gamma \in S$ if and only if $-\gamma \in S$; such a curve is called real. Secondly in coordinates $(w, z)$ defined above, $S$ can be shown [7] to be the zero set of a polynomial of the form

$$
p(w, z)=w^{k}+a_{1}(z) w^{k-1}+\ldots+a_{k}(z),
$$

where $a_{j}(z)$ is a polynomial of degree $2 j$ in $z$. A curve of this form is called a curve of degree $2 k$.

For $\mathrm{SU}(2)$ and $k=1$ there is the well-known Prasad-Sommerfield monopole whose only parameter is its position (zero of the Higgs field). The spectral curve of this monopole is the collection of all lines through its centre.

\section{The Spectral Curves}

For the general group $K$ the spectral curves can be obtained from the fundamental representations of $K$.

We recall $[1,9]$ that for a group $K$ with maximal torus $T$ the complexified Lie algebra of $K$ decomposes as $L K^{c}=L T^{c} \bigoplus_{\alpha \in \Omega} L_{\alpha}$, where $\Omega \subset L T^{*}$ is the set of roots of $K$. If a regular element $X \in L T$ is fixed, we can divide the roots $\alpha$ into positive and negative depending on the sign of $\alpha(X)$. Amongst the positive roots, there is a distinguished set $\left\{\alpha_{1}, \ldots, \alpha_{r}\right\}$ of simple roots which form a basis for $L T^{*}$. The weights $\Lambda$ are the set of $\mu \in L T^{*}$ such that $2 \frac{\langle\mu, \alpha\rangle}{\langle\alpha, \alpha\rangle}$ is an integer for all $\alpha \in \Omega$, where 
$\left\langle>\right.$ is the Killing form. A weight $\mu$ is called dominant if $2 \frac{\left\langle\mu, \alpha_{i}\right\rangle}{\left\langle\alpha_{i}, \alpha_{i}\right\rangle} \geqq 0$ for every simple root $\alpha_{i}$. The lattice of weights has as generator the fundamental weights $\mu_{i}$ defined by $2 \frac{\left\langle\mu_{i}, \alpha_{j}\right\rangle}{\left\langle\alpha_{j}, \alpha_{j}\right\rangle}=\delta_{i j}, \forall i, j=1 \ldots r$. Then $\mu \in \Lambda$ is dominant if and only if $\mu=\sum_{i=1}^{n} n_{i} \mu_{i}$ for positive (integral) $n_{i}$.

Now if $V$ is an irreducible representation of $K, V$ decomposes into weight spaces $V=\bigoplus_{\mu \in \pi(V)} V(\mu)$, where $\pi(V) \subset \Lambda$. If we partially order the weights by $\lambda \geqq \mu$ if $\lambda-\mu$ is a sum of positive roots, then $\pi(V)$ has a unique highest weight $\lambda$, which is dominant and $\operatorname{dim} V(\lambda)=1$. Conversely if $\lambda$ is a dominant weight there is an irreducible representation $V_{\lambda}$ with highest weight $\lambda$.

For example, in $\mathrm{SU}(n), L T$ is the collection of diagonal matrices $X=i \operatorname{diag}\left(\lambda_{1}, \ldots, \lambda_{n}\right)$ and $\sum \lambda_{i}=0$. If $x_{i} \in L T^{*}$ is the $i^{\text {th }}$ projection, so that $x_{i}(X)=\lambda_{i}$, then the roots are $x_{i}-x_{j}, i \neq j$. Conventionally, the positive roots are $x_{i}-x_{j}$ for $i<j$, and then the simple roots are $\left\{x_{1}-x_{2}, \ldots, x_{n-1}-x_{n}\right\}$. The fundamental weights are $\left\{x_{1}, x_{1}+x_{2}, \ldots, x_{1}+x_{2}+\ldots+x_{n-1}\right\}$ and the fundamental representations are $\mathbb{C}^{n}, \Lambda^{2} \mathbb{C}^{n}, \ldots, \Lambda^{n-1} \mathbb{C}^{n}$.

Using these results, let $K \rightarrow \operatorname{SU}\left(V_{\lambda}\right)$ be a representation of $K$ with highest weight $\lambda$. Then, as for $\mathrm{SU}(2)$, we can consider the vector space, $E_{\lambda}(\gamma)$ of solutions of

$$
\left(D_{t}-i \Phi\right) v(t)=0
$$

along the line $\gamma$, where $v(t)$ takes values in $V_{\lambda}$. If $\langle$,$\rangle is the inner product on V_{\lambda}$ and $w(t) \in E_{\lambda}(\gamma)$ and $v(t) \in E_{\lambda}(-\gamma)$, we have

$$
\begin{aligned}
\frac{d}{d t}\langle w(t), v(t)\rangle & =\left\langle D_{t} v, w\right\rangle+\left\langle v, D_{t} w\right\rangle \\
& =\langle i \Phi v, w\rangle+\langle v,-i \Phi w\rangle \\
& =\left\langle v,-i \Phi^{*} w-i \Phi w\right\rangle \\
& =0,
\end{aligned}
$$

as $\Phi^{*}+\Phi=0$ in $\operatorname{LSU}\left(V_{\lambda}\right)$. So there is a well defined pairing

$$
\langle,\rangle: E_{\lambda}(\gamma) \times E_{\lambda}(-\gamma) \rightarrow \mathbb{C} .
$$

Applying the same result [4] as for SU(2), we see that for each eigenvector $e(\mu) \in V_{\lambda}(\mu)$ there is some $v(t) \in E_{\lambda}(\gamma)$ such that

$$
v(t) t^{-\mu\left({ }^{*} F^{\infty}\right) / 2} e^{\mu\left(\Phi^{\infty}\right) t} \rightarrow e(\mu)
$$

as $t \rightarrow+\infty$.

So, in particular, we can define the one dimensional subspace

$$
E_{\lambda}^{+}(\gamma)=\left\{v \in E(\gamma) \mid\left\|v(t) t^{-\lambda\left({ }^{*} F^{\infty}\right) / 2} e^{\lambda\left(\Phi^{\infty}\right) t}\right\| \text { is bounded as } t \rightarrow+\infty\right\} .
$$

Similarly we can define the $\operatorname{dim}_{\lambda} V-1$ dimensional space

$$
\begin{aligned}
E_{\lambda}^{-}(\gamma)= & \left\{v \in E(\gamma) \mid\left\|v(t) t^{\left.-\mu{ }^{*} F^{\infty}\right) / 2} e^{\mu\left(\Phi^{\infty}\right) t}\right\|\right. \\
& \text { is bounded as } t \rightarrow-\infty \text { for all } \mu<\lambda\} .
\end{aligned}
$$


Then, by considering the decay rates, we have

$$
\left\langle E_{\lambda}^{+}(\gamma), E_{\lambda}^{-}(-\gamma)\right\rangle=0 .
$$

So we can define the real algebraic curve

$$
S_{\lambda}=\left\{\gamma \mid E_{\lambda}^{+}(\gamma) \subset E_{\lambda}^{-}(\gamma)\right\}
$$

the spectral curve associated to the dominant weight $\lambda$.

Now if $\lambda$ is a dominant weight then $\lambda=\sum n_{i} \mu_{i}$, where the $n_{i}$ are non-negative integers and the $\mu_{i}$ are the fundamental weights. So we define $S_{i}=S_{\mu_{i}}$ the $i^{\text {th }}$ spectral curve of the monopole. Then we can show that

$$
S_{\lambda}=\sum_{i=1}^{r} n_{i} S_{i}
$$

where this notation means that if $p_{\lambda}(w, z), p_{i}(w, z)$ are the polynomials defining $S_{\lambda}$ and $S_{i}$ then

$$
p_{\lambda}(w, z)=\sum_{j=1}^{r}\left(p_{i}(w, z)\right)^{n_{i}} .
$$

For a general monopole the topological weights can be defined from ${ }^{*} F^{\infty}$ $[6,13]$. The $i^{\text {th }}$ topological weight is defined to be

$$
m_{i}=\mu_{i}\left(2^{*} F^{\infty}\right) \text {. }
$$

Then we can show that $S_{i}$ is a real, algebraic curve of degree $2 m_{i}$, so that $S_{\lambda}$ is a real algebraic curve of degree $4 \lambda\left({ }^{*} F^{\infty}\right)$.

We note that this result demonstrates that the topological weights of a monopole are all non-negative. From the work of Taubes [13] there are monopoles for each set of non-negative topological weights.

For $\mathrm{SU}(3),{ }^{*} F^{\infty}$ must lie in the shaded region below:

Fig. 1

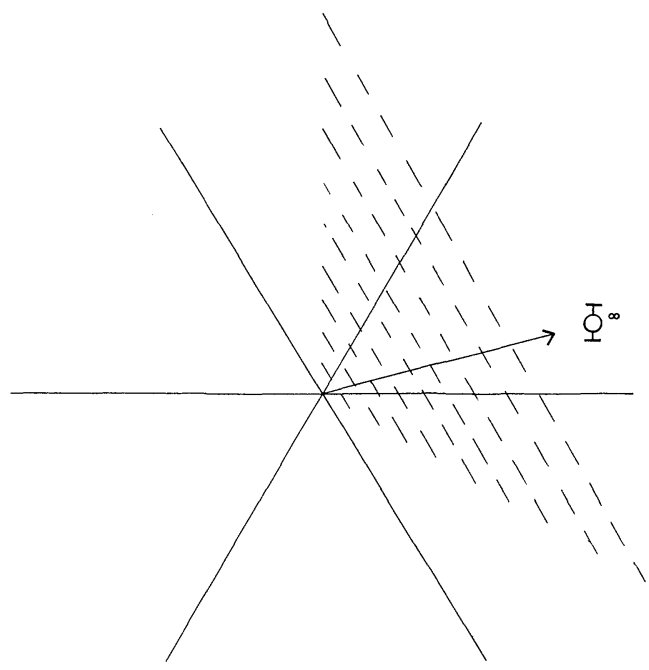


Weinberg [15] has introduced the notion of a fundamental monopole, which is an embedding of an $\mathrm{SU}(2)$ Prasad-Sommerfield monopole into $K$ along a simple root direction. For these, one of the spectral curves is the collection of the lines through the monopole's center and the others are empty sets.

\section{The Classical Groups}

For each of the classical groups, $\mathrm{SU}(n), \mathrm{Sp}(n), \mathrm{SO}(2 n)$, and $\mathrm{SO}(2 n+1)$ we have standard representations. Using these we can apply the twistor construction and interpret the result in terms of the fundamental representations. We give some details for $\mathrm{SU}(n)$.

From (11) the asymptotic expansion of the Higgs field along a line $\gamma$ is

$$
\Phi=i \operatorname{diag}\left(\lambda_{1}, \ldots, \lambda_{n}\right)-\frac{i}{2 r} \operatorname{diag}\left(k_{1}, \ldots, k_{n}\right)+O\left(\frac{1}{r^{2}}\right),
$$

with $\lambda_{1}+\ldots+\lambda_{n}=0=k_{1}+\ldots+k_{n}$ and $\lambda_{1}>\ldots>\lambda_{n}$.

Then we define the space $E(\gamma)$ as usual and the subspaces $E_{i}^{+}(\gamma)$ by

$$
E_{i}^{+}(\gamma)=\left\{v(t) \in E(\gamma) \mid\left\|v(t) t^{-k_{z} / 2} e^{+\lambda_{i} t}\right\| \text { is bounded as } t \rightarrow+\infty\right\},
$$

and the analogous space $E_{i}^{-}(\gamma)$ by

$$
E_{n-i+1}^{-}(\gamma)=\left\{v(t) \in E(\gamma) \mid\left\|v(t) t^{-k_{i} / 2} e^{\lambda_{i} t}\right\| \text { is bounded as } t \rightarrow-\infty\right\} .
$$

Again we have a pairing and now

$$
\left\langle E_{i}^{+}(\gamma), E_{n-i}^{-}(-\gamma)\right\rangle=0 \text { and }\left\langle E_{i}^{-}(\gamma), E_{n-i}^{+}(-\gamma)\right\rangle=0 .
$$

Using these we can define

$$
T_{i}=\left\{\gamma \mid E_{i}^{+}(\gamma) \cap E_{n-i}^{-}(\gamma) \neq 0\right\} .
$$

Alternatively $T_{i}$ is the collection of points $\gamma$ where

$$
\Lambda^{i} E_{i}^{+}(\gamma) \rightarrow \Lambda^{i}\left(E / E_{n-1}^{-}(\gamma)\right)
$$

vanishes. If we make the appropriate identifications it is not difficult to see that $S_{i}=T_{i}$, the $i^{\text {th }}$ spectral curve. These curves must satisfy a constraint on the intersections $S_{i} \cap S_{j}$ when $i$ and $j$ are joined on the Dynkin diagram (see Sect. 5).

Now in [7] Hitchin recovered the monopole by building up the (2-dimensional) bundle $E$ from a 1-dimensional bundle $L^{+}$using the spectral curve. In a similar way we can use the whole flag $E_{1}^{+} \subset E_{2}^{+} \subset \ldots \subset E$ and build up the bundle in a series of $n-1$ steps using the $i^{\text {th }}$ spectral curve at the $i^{\text {th }}$ stage. To make this procedure work we need the technical requirement on the spectral curves that $S_{i} \cap S_{j}$ is finite whenever the $i^{\text {th }}$ and $j^{\text {th }}$ simple root are joined on the Dynkin diagram.

For each of the classical groups a similar procedure works and under the same conditions the spectral curves determine the monopole. In the case of the exceptional groups the adjoint representation is the obvious one to use and, although complicated, this seems to work in principle. 
Recently Nahm [11] has extended his use of the ADHM method of constructing monopoles to the $\mathrm{SU}(n)$ case and produced $n-1$ algebraic curves associated to each monopole. It would seem plausible that these are the spectral curves but we have not verified this yet.

\section{Further Details}

We present some more details using the notation of [7]. The twistor correspondence there can be generalized as follows. If we have a monopole for $P\left(\mathbb{R}^{3}, K\right)$ with reduction at infinity to $T$ a maximal torus there is a natural holomorphic principal bundle $Q\left(T \mathbb{P}_{1}, G\right)$, where $G$ is the complexification of $K$. If $\sigma: G \rightarrow G$ is an antiholomorphic involution fixing $K$ and $B$ a Borel subgroup such that $K \cap B=T$, then $Q$ has a "real structure" $\sigma: Q \rightarrow Q$ covering $\tau$ on $T \mathbb{P}_{1}$ and satisfying $\sigma(q g)=\sigma(q) \sigma(g) \forall q \in Q g \in G$, and two reductions, $R^{+}$and $R^{-}$to $B$ and $\sigma(B)$ respectively, satisfying $\sigma\left(R^{+}\right)=R^{-}$and

$$
R^{ \pm}(\lambda) \cong L^{\lambda\left(\phi^{\infty}\right)}\left(\mp 2 \lambda\left({ }^{*} F^{\infty}\right)\right)
$$

where $R^{+}(\lambda)$ is the associated line bundle induced by the weight $\lambda$.

Then using these, and a representation $V_{\lambda}=\bigoplus_{\mu \in \pi(\lambda)} V_{\lambda}(\mu)$, where $\pi(\lambda)$ are the weights of the representation; $S_{\lambda}$ is defined by

$$
\phi_{\lambda}: R^{+}\left(V_{\lambda}(\lambda)\right) \rightarrow R^{-}\left(V_{\lambda}\right) / R^{-}\left(\bigoplus_{\mu<\lambda} V_{\lambda}(\mu)\right) .
$$

Here if $P(M, G)$ is a principal bundle and $G$ acts on $F$ on the left, we let $P(X)$ $=P \times{ }_{G} F$ be the standard associated bundle. Using (34) we see that $\phi_{\lambda} \in H^{0}\left(T \mathbb{P}_{1}, O\left(4 \lambda\left({ }^{*} F^{\infty}\right)\right)\right)$.

If we specialize to the case of $\lambda_{i}$ a fundamental weight, and let $V_{\lambda_{i}}=V_{i}$, then in the natural ordering the next weight after $\lambda_{i}$ is $\lambda_{i}-\alpha_{i}$ with multiplicity 1 . So the following diagram can be defined

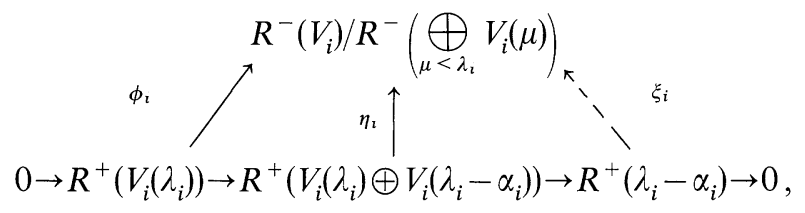

where $-\rightarrow$ indicates $\xi_{i}$ is defined only where $\phi_{i}$ vanishes, that is on $S_{i}$.

We can also obtain the spectral curves by considering the induced sections $R^{-}$of $Q(G / B) \cong R^{+}(G / B)$, the flag bundle. Now $G / B$ has a cell decomposition stable under $B$ so each fibre of $R^{+}(G / B)$ has a cell decomposition $[2,3]$. The cells of $G / B$ are labelled by the elements of the Weyl group and we need to know the following: (1) there is one cell of dimension $G / B$, (2) there are $r$ cells $C_{1}, \ldots, C_{r}$ of codimension 1 corresponding to the reflections $\sigma_{i}$ in the hyperplanes orthogonal to $\alpha_{i},(3)$ if $\left\langle\alpha_{i}, \alpha_{j}\right\rangle=0$, then $\bar{C}_{i} \cap \bar{C}_{j}$ contains one cell $C_{i j}$ (labelled by $\sigma_{i} \sigma_{j}=\sigma_{j} \sigma_{i}$ ), and (4) if $\left\langle\alpha_{i}, \alpha_{j}\right\rangle \neq 0$, then $\bar{C}_{i} \cap \bar{C}_{j}$ contains two cells $C_{i j}$ and $C_{j i}$. Then if we look at the intersection of $\bar{C}_{i}$ and the section $R^{-}$and project onto $T \mathbb{P}_{1}$, we obtain $S_{i}$. Furthermore $S_{i} \cap S_{j}$ is the union of two naturally defined subsets $S_{i j}$ 
and $S_{j i}$. Then the $\xi_{i}$ defined above vanishes on the union of the $S_{i j}$ for $\alpha_{j}$ joined to $\alpha_{i}$ on the Dynkin diagram.

As an example for SU(3) we have two curves, $S_{1}$ where $E_{1}^{+} \subset E_{2}^{-}$, and $S_{2}$ where $E_{1}^{-} \subset E_{2}^{+}$, and then $S_{12}$ is where $E_{1}^{+}=E_{1}^{-}$, and $S_{21}$ where $E_{2}^{-}=E_{2}^{+}$.

Then it is the $S_{i}$ and $\xi_{i}$, or the $S_{i}$ and the division $S_{i} \cap S_{j}=S_{i j} \cup S_{j i}$, which determine the monopole.

\section{Conclusion}

The spectral curves provide some interesting insight into the properties of the monopole solution for the case of maximal symmetry breaking. When there are some coincident eigenvalues in the Higgs field at infinity it is not yet clear if the spectral curves should be expected to determine the monopole.

It would be interesting also to understand why the Dynkin diagram appears in the form it does and how this manifests itself in Nahm's work.

Acknowledgement. The author would like to thank Professor M. F. Atiyah whose help and inspiration underlies all of this work, Dr. N. Hitchin for helpful discussions and the Association of Commonwealth Universities for financial support.

\section{References}

1. Adams, J.F.: Lectures on Lie groups. New York, Amsterdam: Benjamin 1967

2. Atiyah, M.F.: Convexity and commuting Hamiltonians. Bull. London Math. Soc. 14, 1-15 (1982)

3. Bernstein, I.N., Gel'fand, I.M., Gel'fand, S.I.: Schubert cells and cohomology of the spaces $G / P$. Russ. Math. Surv. 28, 1-26 (1973)

4. Coddington, E.A., Levinson, N.: Theory of ordinary differential equations. New York, Toronto, London: McGraw-Hill 1955

5. Corrigan, E., Goddard, P.: An n-monopole solution with $4 n-1$ degrees of freedom. Commun. Math. Phys. 80, 575-587 (1981)

6. Goddard, P., Nuyts, J., Olive, D. : Gauge theories and magnetic charge. Nucl. Phys. B125, 1 (1977)

7. Hitchin, N.: Monopoles and geodesics. Commun. Math. Phys. 83, 579 (1982)

8. Hitchin, N.: On the construction of monopoles. Commun. Math. Phys. 89, 145-190 (1983)

9. Humphreys, J.E.: Introduction to Lie algebras and representation theory. Berlin, Heidelberg, New York: Springer 1972 (2nd edn.)

10. Jaffe, A., Taubes, C.: Vortices and monopoles. Boston: Birkhäuser 1980

11. Nahm, W.: The algebraic geometry of multimonopoles. Bonn Preprint, HE-82-30

12. Prasad, M.K.: Yang-Mills-Higgs monopole solutions of arbitrary topological charge. Commun. Math. Phys. 80, 137-149 (1981)

13. Taubes, C.H.: The existence of multi-monopole solutions to the non-abelian, Yang-Mills-Higgs equations for arbitrary simple gauge groups. Commun. Math. Phys. 80, 343-367 (1981)

14. Ward, R.S.: A Yang-Higgs monopole of charge 2. Commun. Math. Phys. 79, 317-325 (1981)

15. Weinberg, E.: Fundamental monopoles and multimonopole solutions for arbitrary simple gauge groups. Nucl. Phys. B 167, 500 (1980)

Communicated by A. Jaffe

Received March 10, 1983

Note added in proof. It has not yet been shown that the spectral curves determine the $\mathrm{SO}(2 n+1)$ monopole or the monopoles for the exceptional groups. 
